\title{
Weitere Unstetigförderer
}

\author{
Ludger Overmeyer
}

\subsection{Elektrohängebahn}

Elektrohängebahnen sind schienengeführte, flurfrei angeordnete Transportsysteme mit einzeln angetriebenen Fahrzeugen [1]. Hierdurch ist ein reversierbarer Betrieb möglich. Sie dienen zur Verbindung einer oder mehrerer Quellen mit einer oder mehreren Senken sowie zum Puffern und Sortieren von Stückgütern.

Elektrohängebahnen bestehen aus den Systemkomponenten Schiene, vertikale Umsetzeinrichtungen, Fahrzeuge, Lastaufnahmeeinrichtungen, Energiezufuhr und Steuerung.

Durch den Einsatz von horizontalen und vertikalen Kurven bis $90^{\circ}$ sowie Weichen sind beliebige Fahrkurse realisierbar. Vertikale Umsetzeinrichtungen verbinden Laufschienen auf verschiedenen Transportebenen; maximale Hubgeschwindigkeiten liegen bei ca. 2,5 m/s.

In Abhängigkeit von der Fahrzeug- und Schienenausführung unterscheidet man zwischen Außen-, Innen- und Obenläufern. Die Übertragung der Antriebskraft vom Fahrzeug auf die Fahrschiene erfolgt im Regelfall durch Reibschluss, bei hohen Steigungen auch durch Formschluss.
Die Fahrzeuge bestehen aus einem angetriebenen sowie bei höheren Traglasten aus einem zusätzlichen nicht angetriebenen Teil. Diese sind durch eine Traverse gekoppelt. Typische Traglasten bei horizontalem Streckenverlauf betragen $250 \mathrm{~kg}$ bis $750 \mathrm{~kg}$. Der Antrieb erfolgt meist durch Drehstromasynchron- oder Gleichstrommotoren. Die Übertragung der elektrischen Versorgungsspannung erfolgt mittels Stromschienen oder Schleppkabeln. Alternativ kann der Antrieb auch manuell, durch Schwerkraft, pneumatisch oder hydraulisch erfolgen. Die maximale Fahrgeschwindigkeit beträgt bei automatisch gesteuerten Hängebahnen ca. $3,0 \mathrm{~m} / \mathrm{s}$.

Zur Steuerung der Elektrohängebahnen dienen Handsteuerungen sowie Speicherprogrammierbare Steuerungen (SPS). Eine Einbindung in Fertigungszellen ist möglich.

\section{Literatur}

\section{Spezielle Literatur}

1. VDI-Richtlinie 3643: Elektro-Hängebahn-Obenläufer, Traglastbereich $500 \mathrm{~kg}$ Anforderungsprofil an ein kompatibles System. VDI-Verlag, Düsseldorf (1998)

L. Overmeyer $(\square)$

Leibniz Universität Hannover

Garbsen, Deutschland

E-Mail: ita@ita.uni-hannover.de 